\title{
Some Proper Methods for Optimization in Robotics
}

\author{
Olaru D. Adrian, Olaru A. Serban, and Mihai C. Niculae
}

\begin{abstract}
Optimization in Robotics are applied to find the best solution for increasing the $3 \mathrm{D}$ space precision, reduce the vibrations, choose the optimal applied base point of the robots to minimize the time of application, finding constructive or functional parameters that ensure reduced energy consumption, reduce the vibration and increase the stability of the movement, choose the optimal variation of the moments in all joints, finally to optimize the dynamic behavior.

In the work was developed one proper algorithm to obtain, iteratively the extreme performances to the 3D space trajectory without vibration in the desirable field of the Fourier spectrum. The research show how can be increased the accuracy of the internal coordinate by solving the inverse kinematics with proper method with the goal to touch the extreme precision better than $0.001 \mathrm{~mm}$.
\end{abstract}

Index Terms-Forward kinematics, Inverse kinematics, fourier generator, inverse kinematics, neural network, smart damper system.

\section{Generality ABOUT THE OPTIMIZATION WORK}

Optimization means finding the best solution for a problem under given circumstances. Mathematical optimization means that the problem to have solving must be respect one objective function, the constraints for all imposed parameters and one iterative algorithm that was validated by the experimental assisted research.

Many problems in robotics require optimization of multiple conflicting criteria, such as the speed of a system and its energy efficiency, or precision and stability.

The optimization problem can be single or multi- objective problem. In the multi-objective optimization (MOO) problem, these multiple objectives are treated independent between them. Instead of a single optimum, this gives rise to a set of Pareto optimal solutions, termed the Pareto set [1]-[7].

Another important consideration when optimizing the performance of a robotic system is that the optimization work can be expensivesuch as time of assisted research, financial cost, or use of computational resources. In this work we consider a single objective function for different optimization proposed ways. In the expensive MOO case, the goal is to reduce the number of experiments needed to find the Pareto solutions. Methods such as descent gradient method or genetic algorithms are not designed to limit the number of objective functions.

When it comes to design optimization, Papalambros et al. give the following definition in [8]: "Informally, but

Manuscript received on March 30, 2017; revised May 23, 2017.

A. D. Olaru is with the University Politehnica of Bucharest, RO 0060042 Romania (e-mail: aolaru_51@ymail.com).

S. A. Olaruis is with RomSys Mechatronics Private Company, Bucharest, Ro 013713 Romania (e-mail: serban1978@yahoo.com).

N. C. Mihai is with TechnoAccord Mechatronics Private Company, Montreal, CA 4170, Canada (e-mail: mniculae@yahoo.com). rigorously, we can say that design optimization involves: the selection of a set of variables to describe the design alternatives; the selection of an objective (criterion), expressed in terms of the design variables, which we seek to minimize or maximize; the determination of a set of constraints, expressed in terms of the design variables, which must be satisfied by an desirable or acceptable design; the determination of a set of values for the design variables, which minimize (or maximize) the objective, while satisfying all the constraints." Design variables are in this work also called optimization variables when they are used in optimization formulations.

In any cases of optimization work the required steps are the following: establishing all desirable and needed optimizing ways; define the optimization functions conformity with the needed results; establishing the objective function's values and constraints violation;establishing the optimization mathematical algorithm; define detailed system parameters model; make the simulation procedure and obtain the results characteristics; comparing the results with the required performances and adjust iteratively the algorithm to touch the imposed target with respect strictly the objective function.

Many design methods, which are applicable to robot design, exist in academic literature.

The concept of manipulability was introduced by Yoshikawa [9] as a means to measure the ability of robotic system in positioning and orienting end effecters. Asada [10] introduced the generalized inertia ellipsoid as a tool to measure the capability of changing the velocity of the end effecter. Furthermore, Graettinger and Krogh [11] developed the acceleration radius like a optimized method. The acceleration radius is a global generalization of the local measures of the dynamic responsiveness proposed by other researchers such as Yoshikwa [9]. The acceleration radius is a minimum limit of the acceleration'smagnitude that can be achieved at the end effector from any state (joint position and velocity) in the working space. Furthermore, Bowling presents in [12] a whole analysis of robotic dynamic performance.Ma and Angeles [13] showed how the architecture of a manipulator is optimized under dynamic isotropy conditions. Here, the design strategy is to render the generalized inertia matrix of a manipulator as close to dynamic isotropy and hence achieve optimum dynamic performance. In recent years, Angeles has put forward methods which focus on the kineto static optimization of manipulators [14]. These methods reveals on the minimization of a condition number of the Jacobian matrix over the architectural parameters and the posture variables of the manipulator. The minimization of the condition number of the Jacobian has been put forward by others [15], but Angeles introduced the characteristic length in order to 
limited the Jacobian matrices. In [16], the concept is revisited and put forward from a different point of view. The concept of homogeneous space is introduced in order to relieve the designer from the concept of characteristic length. A more straightforward kinematic measure, normally used by industrial robot manufacturers, is based on the maximum reach (see Fig. 1) of a robot. Other kinematic performance measures used by industrial manufacturers that affect the shape of the workspace is the so called stroke. The stroke is defined as the offset between maximum reach and minimum reach of the end effector of a robot.

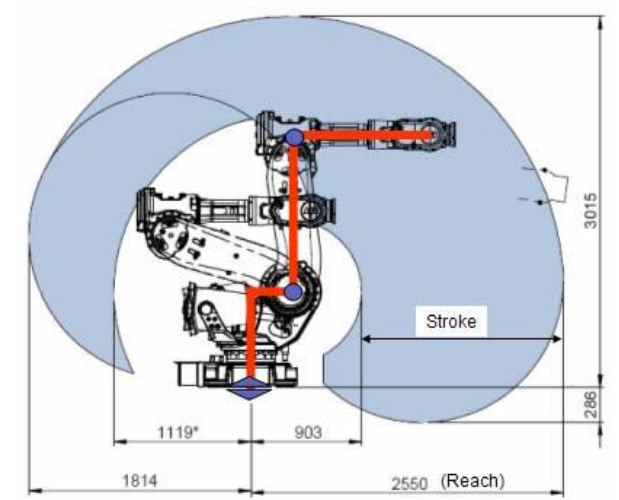

Fig. 1. Shape, reach, and stroke of the workspace of an ABB IRB6640-185/2.8 robot.

The robot configuration, the structure and the drive are designed based on the cycle time (time required to execute working cycles) or about the speed and acceleration requirements. The mass data is obtained based on an initial design of the application.

\section{SOME AlgORIthMS FOR OPTIMIZATION}

In the optimization work the steps that must be solve are the followings: -define the object function; -define the all imposed constraints by the physical application and for the robot's designed structure; -make and apply the iterative algorithm; -adjust the algorithm before touch the convergence process.

\section{A. Optimization the Position of the Base Point}

In this kind of application will be necessary to find the better position of the application base robot's point in the $3 \mathrm{D}$ space to obtain the minimum time of robot work. The algorithm contents the inverse kinematic solving of the used robot, the forward kinematics and neural network to obtain the internal coordinate that assures the touching space target with one precision more than $0.001 \mathrm{~mm}$. The application points are finding in one cube that follows the constraints conditions. The proposed algorithm Pseudo- Inverse Jacobian- Matrix- Method with Sigmoid- BipolarHyperbolic- Tangent- Neural- Network with Time- Delay and Recurrent- Links (PIJMM- SBHTNN- TDRL)will be applied for all application points of robot [17- 19], points that will be obtainedby interpolation between the constraints limits of the base point of the robot. After that, will be resulted the internal coordinates that assured also the extreme precision and the better position of the robot's base. The objective function of optimization problem is calculate for all possible application points $p_{i}$, represents the minimum time of motion between points $\mathrm{P}_{1}$ and $\mathrm{P}_{\mathrm{i}}$ of the proposed application, when the application is multi robots and the movements of all joints are successively:

(1)and the constraints:

$\mathrm{q}_{\mathrm{i} \min }<\mathrm{q}_{\mathrm{i}}<\mathrm{q}_{\mathrm{i} \max }$;

$$
f=\left[x, y, z\left(q_{i}\right)\right]_{i}-[x, y, z]_{\text {target }} \leq \varepsilon_{a} \quad \mathrm{P}_{\mathrm{i} \min }<\mathrm{P}_{\mathrm{i}}<\mathrm{P}_{\mathrm{i} \cdot \max }^{\prime} ;
$$

or the movements are simultaneously:

$$
F\left(p_{i}\right)=\min \left(\sum_{i=1}^{i} \max \left(\frac{4_{1 i}}{4 i_{i}} \forall p_{i i} \quad \in A_{1}\right)\right)
$$

where: $m$ is the number of DOF; $n$ is the number of robots in application, $i$ - the degree of freedom of each robot, $q_{i^{-}}$the movement in each joint; $q_{i}$ is the velocity in each robot's joints; $p_{i}$ is the possible application points to belong of the acceptable field $A ; P_{i}$ is the end effecter point that must be inside of the working space.

The block schema of the applied algorithm is shown in Fig. 2.

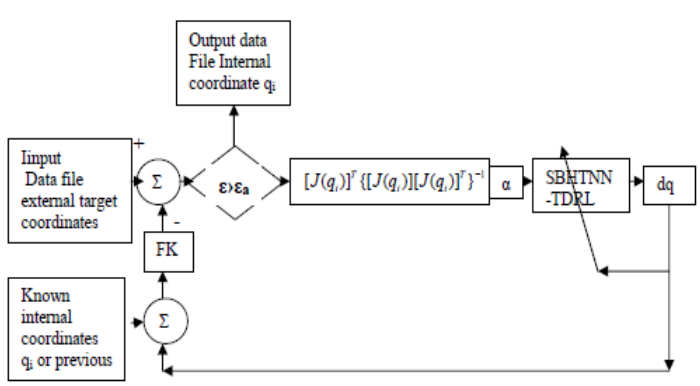

Fig. 2. The block schema of the applied algorithm to find the best solution of the inverse kinematicsPIJMM-BSHTNN-TDRL.

\section{B. Optimization of the Consumption of Energy}

In many cases will be necessary to decrease the consumption of energy, more in the application multi robots. This method of optimization is one unconstrained and constrained method because some of the optimization function parameters is unconstrained.

$$
\left(E\left(q_{i}\right)=\min \iiint_{a p p} F_{x} F_{y} F_{z} d\left(x\left(q_{i}\right) d\left(y\left(q_{i}\right)\right) d\left(z\left(q_{i}\right)\right)\right.\right.
$$

with the constraints:

$$
\begin{aligned}
F_{x \min } & \leq F_{x} \leq F_{x \max } \\
F_{y \text { min }} & \leq F_{y} \leq F_{y \text { max }} \\
F_{z \text { min }} & \leq F_{z} \leq F_{z \max } \\
x_{\text {min }} & \leq x \leq x_{\max } \\
y_{\text {min }} & \leq y \leq y_{\max } \\
z_{\min } & \leq z \leq z_{\max } \\
q_{i \min } & \leq q_{i} \leq q_{i \max }
\end{aligned}
$$

The algorithm in this case is the complex form that must to minimize the consumption of energy by minimize the trajectory and active forces for the successive movements.

\section{Optimization of the 3D Space Trajectory Precision}

In the optimization of the $3 \mathrm{D}$ precision one important role 
have the precision of the solving the inverse kinematics [20]-[26]. The proper method that it is applied was one complex method of Pseudo Inverse Jacobian Matrix Method coupled with Bipolar Sigmoid Hyperbolic Tangent Neural Network with Time Delay and Recurrent Links (PIJMM-BSHTNN-TDRL). The objective function is:

$$
F\left(p_{i}\right)=\min \left(\sum_{i=1}^{i !} \sum_{i=1}^{i n} \frac{q_{i j}}{q_{i j}^{i}} \forall p_{i j} \in A_{i}\right)
$$

The complex algorithm that include the proper Iterative Pseudo Inverse Jacobian Matrix Method (IPIJMM) and proper neural network Bipolar Sigmoid Hyperbolic Tangent Neural Network with Time Delay and Recurrent Links (BSHTNN-TDRL) [27]-[30].

$$
\begin{aligned}
& \left(d q_{i}\right)=\alpha\left[\left(T_{i}\right)-\left(F K\left(q_{i}\right)\right)\right]\left[J\left(q_{i}\right)\right]^{T}\left\{\left[J\left(q_{i}\right)\right]\left[J\left(q_{i}\right)\right]^{T}\right\}^{-1} \\
& \left(q_{j}\right)=\left(q_{i}\right)+\left(d q_{i}\right) \\
& \left.(n)_{1}=[\underbrace{(w w]^{1}}_{p_{1}}+\underbrace{\left(t c g_{1}\right)}_{p_{2}} \cdot\left(\varepsilon_{1}\right)]\left((p)-\left(a_{2}\right)\right)\left((t)-\left(p_{3}\right)+1\right)\right)+\left(\left(b_{1}\right)+\left(\varepsilon_{1}\right)\right) ; \\
& \left(a_{1}\right)=\frac{\left(p_{4}\right)\left(1-e^{-n_{1}}\right)}{1+e^{-n_{1}}} ;\left(\varepsilon_{1}\right)=\left(t_{1}\right)-\left(a_{1}\right) \\
& n_{2}=[w^{2}+\underbrace{t c g_{2}}_{p 5} \cdot \varepsilon_{2}]\left(a_{1}\left(t-p_{6}+1\right)\right)+\left(b_{2}+\varepsilon_{2}\right) ; \\
& a_{2}=\frac{p_{7}\left(1-e^{-n_{2}}\right)}{1+e^{-n_{2}}} ;\left(\varepsilon_{2}\right)=\left(t_{2}\right)-\left(a_{2}\right) \\
& \left(q_{i}\right)=\left(p_{8}\right)\left(\left(a_{2}\right)-\left(\varepsilon_{f}\right)\right) ;\left(\varepsilon_{p o s}\right)=\left(t_{3}\right)-\left(r_{i}\right) ; \\
& \left.\left(n_{3}\right)=[\left[w^{3}\right]+\underbrace{t t c g_{2}}_{p_{5}}) \cdot\left(\varepsilon_{p o s}\right)\right]\left(q_{i}\right)+\left(\left(b_{3}\right)+\left(\varepsilon_{p o s}\right)\right) ; \\
& \left(a_{3}\right)=\frac{\left(p_{9}\right)\left(1-e^{-n_{3}}\right)}{1+e^{-n_{3}}} ;\left(\varepsilon_{f}\right)=\left(t_{2}\right)-\left(a_{3}\right) .
\end{aligned}
$$

\section{Optimization of the Vibration Fourier Spectrum}

Method consists in the decreasing the vibration in one field by impose the desired Fourier spectrum. By this method will be possible to avoid the vibration from the Fourier spectrum that provide from the machine where was positioning the robot. The algorithm contents the Fourier generator, the smart damper, the accelerometers, the smart controller and the proper neural network.

The objective function in this case is:

$$
\varepsilon=F F T\left(x^{\prime \prime}, y^{\prime \prime}, z^{\prime \prime}\right)-F F T_{\text {desired }} \leq \varepsilon_{a}
$$

and the complex algorithm for damper system:

$$
\begin{aligned}
& f=c_{0}\left(x^{\prime}-y^{\prime}\right)+k_{0}(x-y)+k_{1}\left(x-x_{0}\right)+\alpha z \\
& y^{\prime}=\frac{1}{c_{0}+c_{1}}\left[\alpha z+c_{0} x^{\prime}+k_{0}(x-y)\right] \\
& z^{\prime}=-\gamma\left|x^{\prime}-y^{\prime}\right| z|z|^{n-1}-\beta\left(x^{\prime}-y^{\prime}\right)|z|^{n}+\delta\left(x^{\prime}-y^{\prime}\right) \\
& \alpha(i)=\alpha_{3} i^{3}+\alpha_{2} i^{2}+\alpha_{1} i+\alpha_{0} \\
& c_{0}(i)=c_{03} i^{3}+c_{02} i^{2}+c_{01} i+c_{00} \\
& c_{1}(i)=c_{13} i^{3}+c_{12} i^{2}+c_{11} i+c_{10} \\
& k_{0}(i)=k_{03} i^{3}+k_{02} i^{2}+k_{01} i+k_{00} \\
& \delta=\sum \delta_{0 i} \sin \left(2 \pi v_{i}+\phi_{i}\right)
\end{aligned}
$$

and the same for the used neural network where: $x$ and $y$ are the primary, respectively the secondary displacement variables $[\mathrm{m}] ; z$ is the internal history dependency variable of the magneto rheological damper (MRD) [m]; $k_{0}, k_{1}$ are the non linear internal rigidity of the (MRD), $[\mathrm{N} / \mathrm{m}]$ depending of the current intensity $i[\mathrm{~A}] ; c_{0}$ and $c_{1}$ are the internal viscous damping parameters of the (MRD) $[\mathrm{Ns} / \mathrm{m}] ; \alpha$ is the internal parameter whathave non linear evolution and depend on the magnetic variable field (electrical intensity); parameter $\beta$ characterize the gain of increasing of the damping force versus velocity; $x_{0}$ isthe perturbation displacement $[\mathrm{m}] ; \delta$ is the hysteresis parameter.

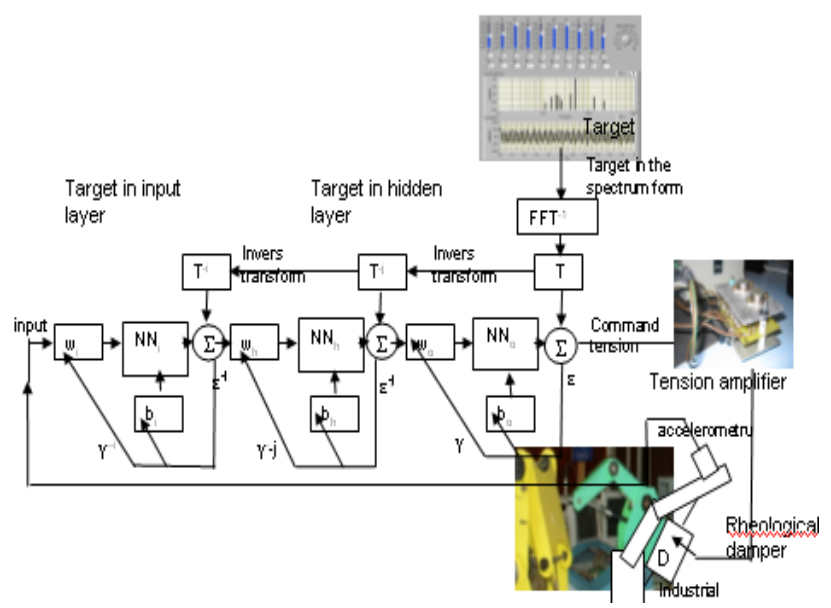

Fig. 3. Block schema for the smart system to optimize the Fourier spectrum of the robot.

\section{E. Optimization of the Torques Characteristics}

The method contents the possibility to find the minimum of variation of the torques of one application, by decreasing the pick of acceleration.Were studied some velocities characteristics with simultaneously movement only during the constant velocities. The objective function is

$$
f=\min M\left(x, y, z, q_{i}, q_{i}^{\prime}, q_{i}^{\prime \prime}\right)
$$
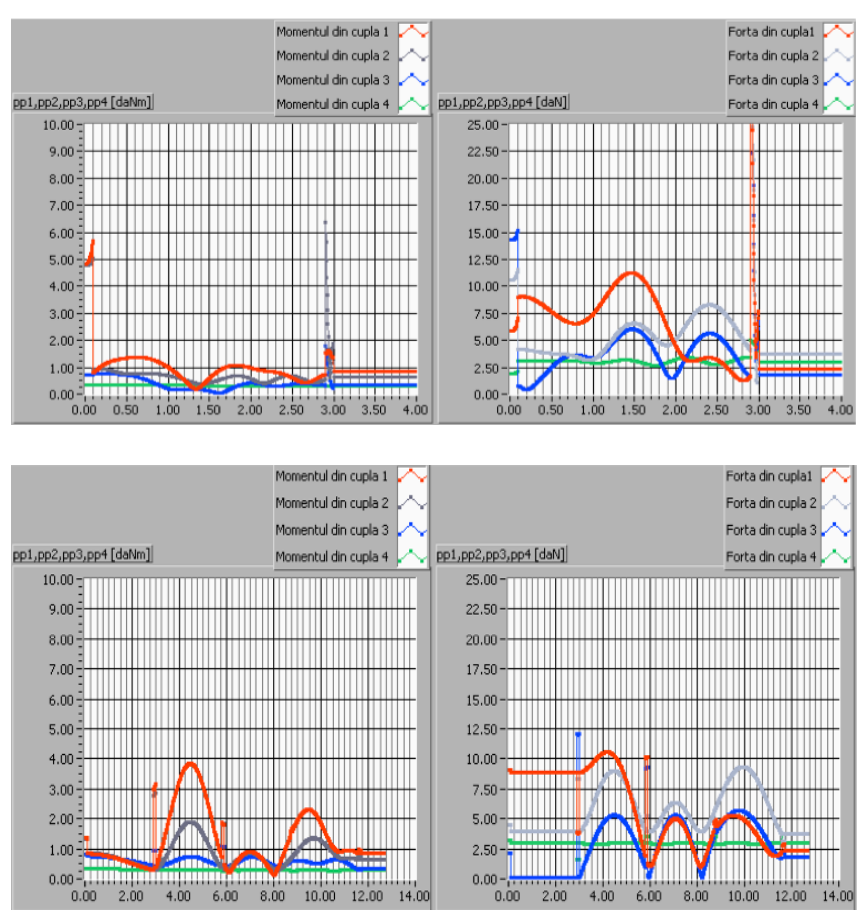


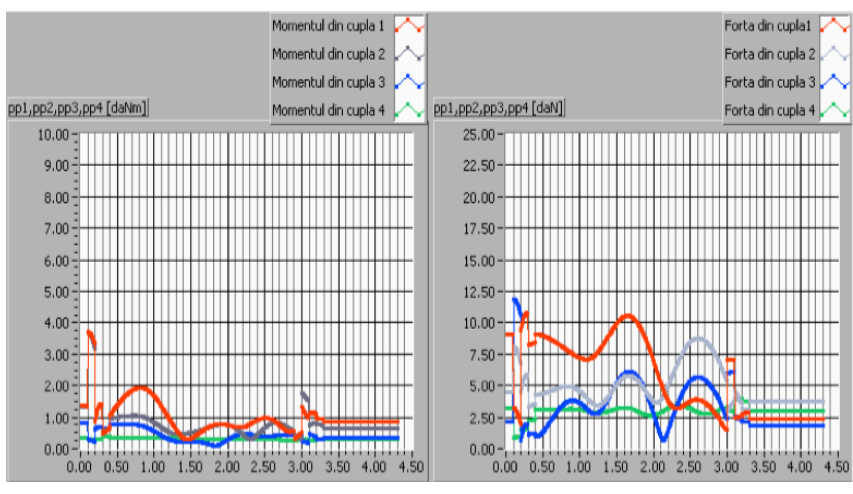

Fig.4. The results of the simulation of the forces and moments in all joints when the velocity characteristics are: successive; successive and simultaneously; simultaneously.

The applied algorithm is:

$$
\begin{gathered}
{\left[K_{g_{i}}^{\prime i}\right]=\left[J_{g_{i}}\right] \cdot[\varepsilon(i)]+\left[\hat{\omega}_{i-1,0}^{i}\right] \cdot\left[J_{g_{i}}\right] \cdot[\omega(i)]} \\
{\left[\begin{array}{c}
K_{g_{i}}^{\prime i} \\
]
\end{array}=\left[J_{g_{i}}\right] \cdot[\varepsilon(i)]\right.} \\
\left(\begin{array}{c}
F^{0} \\
M^{0}
\end{array}\right)=\left[\begin{array}{cc}
z_{u} & 0 \\
0 & z_{u}
\end{array}\right]\left(\begin{array}{c}
D_{0, i}\left(F_{R}^{i}+f\right) \\
D_{0, i} M_{R}^{i}
\end{array}\right)-\operatorname{diag}\left[\operatorname{sign} \frac{v_{u}^{i}}{\left|v_{u}^{i}\right|} m_{u_{i}} \operatorname{sign} \frac{\omega_{u}^{i}}{\left|\omega_{u}^{i}\right|} J_{g_{i}}\right] \cdot\left(\begin{array}{c}
\left(a_{i, 0}^{i}\right)+\left[\left(\widehat{\omega}_{i, 0}^{i}\right]^{2}\left(r_{g_{i}}^{i}\right)\right. \\
\left.\left(\varepsilon_{i, i-1}^{i}\right)+\left[\omega_{i-1,0}^{i}\right]\left(\omega_{i, i-1}^{i}\right)\right)
\end{array}\right)+ \\
+\left[\begin{array}{cc}
z_{u} & 0 \\
0 & z_{u}
\end{array}\right] \cdot\left(\left[G_{i, k}\right]\left(\hat{b}_{i, k}\right)\left(\left(D_{0, i}\left(F_{R}^{i}+f\right)\right)-\operatorname{diag}\left[\operatorname{sign} \frac{v_{u}^{i}}{\left|v_{u}^{i}\right|} m_{u_{i}}\right] \cdot\left[D_{0, i}\right]\left(\left(a_{i, 0}^{i}\right)+\left[\hat{\omega}_{i, 0}^{i}\right]^{2}\left(r_{g_{i}}^{i}\right)\right)\right),\right.
\end{gathered}
$$

where: $r_{i-1}{ }^{0}$ is the position matrix of $i-1$ Cartesian system with respect to the fixed system; $w_{i, 0}{ }^{i}$-absolute dual velocity matrix of $i$ body with respect to the $i$ Cartesian system; $T_{i-1}{ }^{i}$ transfer dual velocity and acceleration matrix from $i$-1 to $i$ Cartesian system; $F$-the active forces matrix in a Cartesian fixed system; $M$ - active moment matrix in a Cartesian fixed system; $z_{u^{-}}$joint-bodies matrix; $D_{i-1}{ }^{i}$ - transfer matrix between $i$-1 and $i$ body; $F_{R^{-}}$resistant forces matrix; $M_{R^{-}}$resistant moments matrix; $m_{i^{-}}$mass matrix of $i$ body; $J_{g i}{ }^{0}$ - inertial tensor matrix of $i$ body; $a_{i, o}{ }^{i}$ - absolute dual acceleration matrix in a $i$ body Cartesian system; $\omega_{i, 0}^{\wedge}{ }^{i}$ non symmetric absolute angular velocity matrix in a $i$ body Cartesian system; $r_{g i}{ }^{i}$ - position of the gravity center matrix in a $i$ body Cartesian system; $\varepsilon_{i, i-1}{ }^{i}$ - angular relative acceleration matrix between $i$ and $i-1$ in a $i$ body Cartesian system; $\omega_{i, i-1}{ }^{i}$ - angular relative velocity between $i$ and $i$-1in a $i$ body Cartesian system; $B^{\wedge}$ modified arm of force matrix; $f$ is the damping force $[\mathrm{N}] ; K_{\mathrm{gi}}^{\mathrm{i}}$ variation of the kinetic moment to the $i$ Cartesian system.

\section{VIRTUAL LABVIEW ${ }^{\mathrm{TM}}$ INSTRUMENTATION}

In the assisted research were used some virtual instruments to find the best solutions at the optimization problem in robotics. Some of these front panel instruments were shown in the following. Each instrument work on-line with possibility to adjust some of the constructive- functional parameters with the final goal to find the better solution to minimize, or maximize the objective functions.
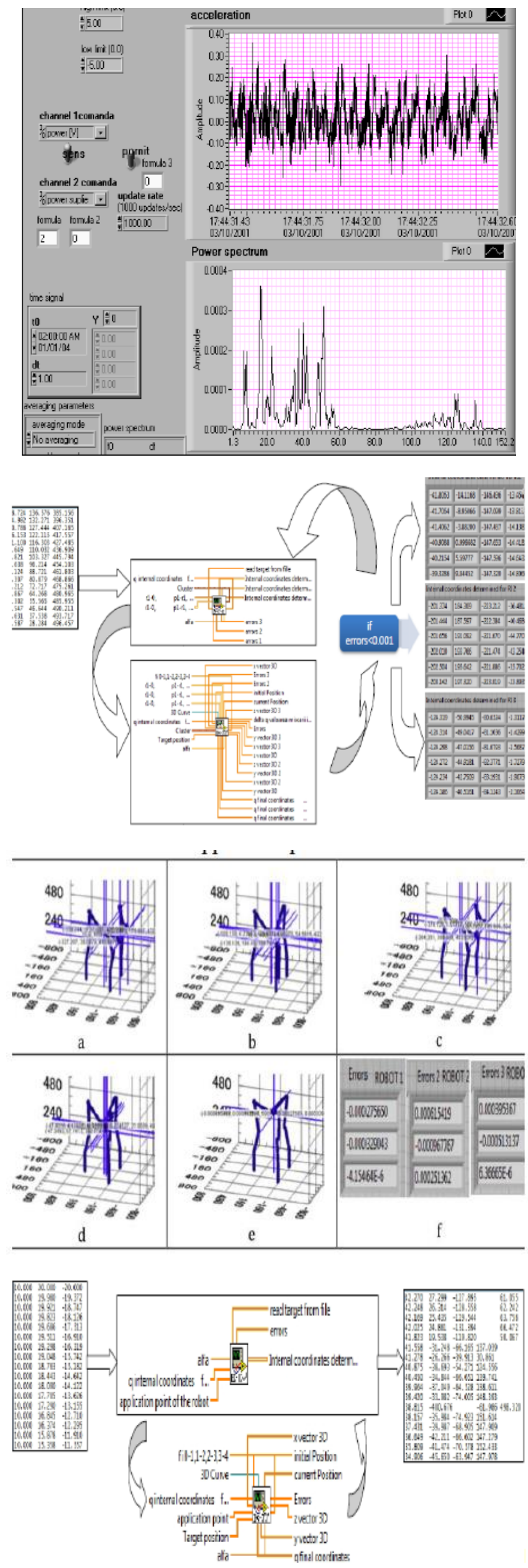

Fig. 5. The virtual LabVIEW instrument for the inverse kinematics problem. 


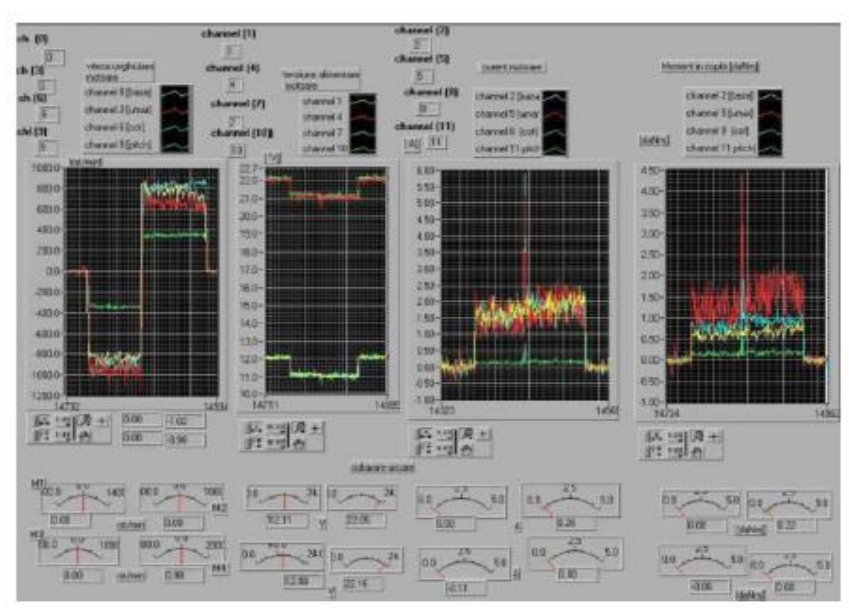

Fig. 6. The virtual LabVIEW instrument for the assisted acquisition of the velocities, acceleration, electrical tension, forces and moments.

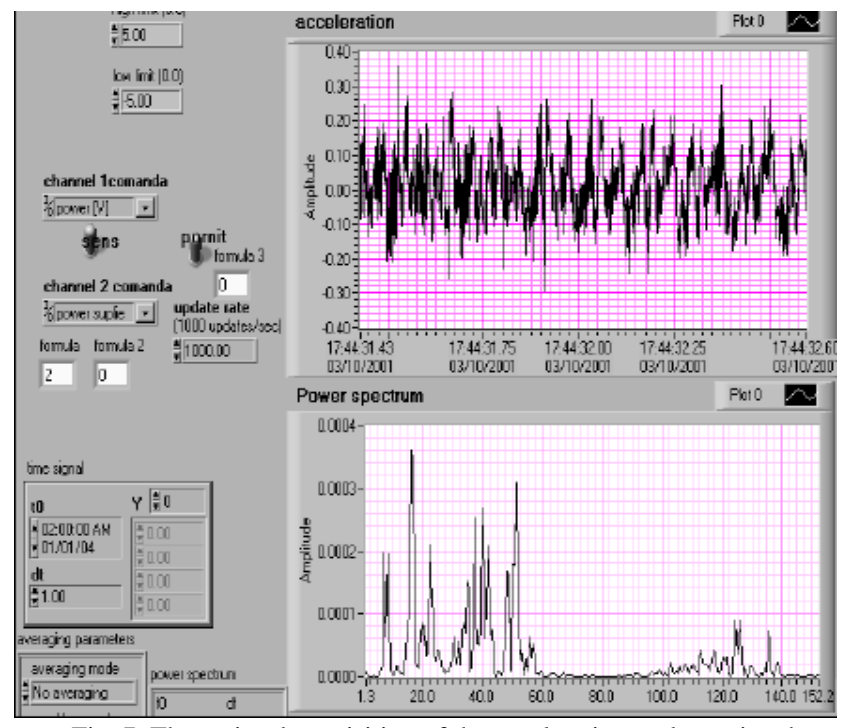

Fig. 7. The assisted acquisition of the acceleration to determine the Fourier spectrum field with minimum of acceleration that will be assured the minimum of the moments.

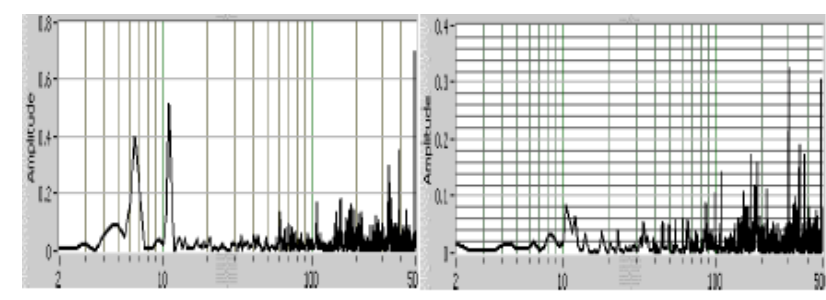

Fig. 8. The assisted research of the Fourier spectrum without and with smart damper in the robot structure.

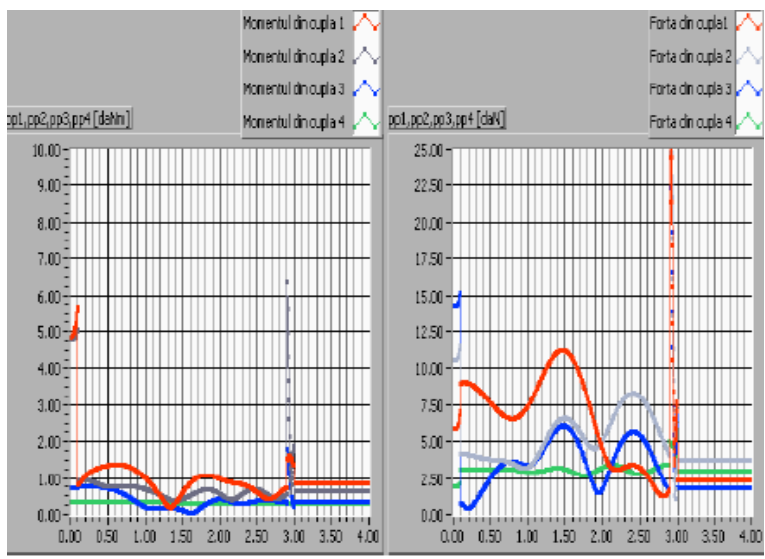

Fig. 9. The numerical simulation of the forces and moments with the proposed algorithm.

\section{Discussing The Results}

The optimization problem is the most complex and important problem in Robotics. Now, in the world the robots with open chain are used like manufacturing systems and for that it is necessary to optimize the dynamic stiffness and compliance by optimize the design of the robot, chose the optimal mass of all bodies, insert the damper smart systems in to the robot's structure to avoid, change or decrease the Fourier vibration spectrum.

After the analyze of the numerical simulation results, using the input data from the experimental setup, we can remark the followings: -the analyze of the position is important to establish the work space and the singularity of some movements; -because all VI-s work on-line will be possible to choose the optimal values for some dimensions of the robot's mechanical parts, body's masses, the velocity command characteristics and what of the joints can work in simultaneously movement without big influences to the forces and moments variations, see Fig. 5 - Fig. 7; -the position analyze offers the possibility to determine the singular points of the movement, the possibility to show the contact or collision with other bodies, can be traced the trajectory of the end effector and see the fuzzy interaction with other space work; -doing the study of the moments and forces characteristics is very important to establish the control low of the magnetorheological damper (control low of the current intensity applied to the damper, what will have the inverse mirror aspect than this), see fig.8; -knowing the forces and moments variation in all joints of the robot's structure assures the correct calculus of the mechanical parts of all robot's modulus, fig.9; -determining the allure of the velocities and accelerations characteristics are important when will be applied some correction of the movements to reduce the forces and moments variation, Fig.7; -the bigger value of the force and moment is in the case of the simultaneously movements and in the case when the first modulus velocity is bigger than others, figs.4; -the pointers forces and moments vectors (3D variation) are important for the dimensioning and calculus of all mechanical parts of the robot's modulus; -apply this method and the VI-s assures to obtain the optimal dynamic behavior, of point of view, for allotment of the movement and pause, fixing the time of movement and pause between the joints movements, and the parameters of the trapezoidal velocity characteristic. The method contents the possibility to find the minimum of variation of the torques between some applications, by decreasing the pick of acceleration.

\section{CONCLUSION}

In the research the $V I$-s approach shortens the automation time significantly and decreases the overall system cost, then increases the efficiency of research as compared with traditional methods or conventional instruments.

LabVIEW's graphical environment is easy to learn, customize and debug the $V I$-s applications as compared with text based programming languages. This enables the researchers to focus on the research project, and then finally accelerate the research. Without the VI-s were not be possible 
to obtain the dynamic behavior of the robots, were not be possible to adjust and validate the complex mathematical matrix model and compare easily the theoretical results with experimental.

\section{ACKNOWLEDGMENT}

The authors tanks to the University Politehnica of Bucharest- Machine and Manufacturing Systems Department, RomSys Company and TechnoAccord Company for technical support to be possible this research.

\section{REFERENCES}

[1] M. Tesch, K. Lipkin, I. Brown, R. Hatton, A. Peck, J. Rembisz, and H. Choset, "Parameterized and scripted gaits for modular snake robots," Advanced Robotics, vol. 23, no. 9, pp. 1131-1158, Jun. 2009.

[2] D. R. Jones, "A taxonomy of global optimization methods based on response surfaces," Journal of Global Optimization, vol. 21, no. 4, pp. 345-383, 2001.

[3] J. L. Cohon, Multiobjective Programming and Planning. Courier Dover Publications, 2004.

[4] V. Coverstone-Carroll, "Optimal multi-objective low-thrust spacecraft trajectories," Computer Methods in Applied Mechanics and Engineering, vol. 186, no. 2-4, pp. 387-402, Jun. 2000.

[5] J. Knowles, "ParEGO: A hybrid algorithm with on-line landscape approximation for expensive multiobjective optimization problems," IEEE Transactions on Evolutionary Computation, vol. 10, no. 1, pp. 50-66, Feb. 2006.

[6] L. Dixon and G. Szego, "The global optimization problem: An introduction," Towards Global Optimization, vol. 2, pp. 1-15, 1978.

[7] A. Z. ilinskas, "A review of statistical models for global optimization," Journal of Global Optimization, vol. 2, no. 2, pp. 145-153, Jun. 1992.

[8] Y. P. Papalambros and J. D. Wilde, Principles of Optimal DesignModeling and Computation, Cambridge University Press, 1988.

[9] T. Yoshikawa, "Manipulability of robotic mechanisms," International Journal of Robotic Research, vol. 4, no. 2, pp. 3-9. 1985.

[10] H. Asada, "A geometrical representation of manipulator dynamics and its application to arm design," Transactions of ASME, Journal of Dynamic Systems., Meas. and Control, vol. 105, pp. 131-135. 1983.

[11] T. Graettinger and B. H. Krogh, "The acceleration radius: A global performance measure for robotics manipulators," Journal of Robotics and Automation, vol. 4, no 1, Feb. 1988.

[12] A. Bowling, "Analysis of robotic manipulator dynamic performance: Acceleration and force capabilities," Ph.D dissertation, Stanford University, 1998.

[13] M. Ou and J. Angeles, "Optimum design of manipulators under dynamic isotropy conditions," in Proc. of IEEE International Conference on Robotics and Automation, vol. 1, pp. 470-475. 1993.

[14] W. A. Kahn and J. Angeles, "The kinetostatic optimization of robotic manipulators: the inverse and the direct problems," Transactions of the ASME Journal of Mechanical Design, vol. 128, no. 1, pp. 168-178, 2006.

[15] J. K. Salisbury and J. J. Craig, "Articulated hands: Force control and kinematic issues," International Journal of Robotics Research, vol. 1, no. 1 , pp. 4-17, 1982.

[16] S. Kirkpatrick, C. D. Gelatt, and M. P. Vecchi, "Optimization by simulated annealing," Science, vol. 220, pp. 671-680, 1983.

[17] L. Ciupitu, Complex analytic curves for industrial robot trajectories, in Proc. of the 3-rd Asian Conference on Robotics and Its Application, Tokyo, Japan, pp. 211-218.

[18] L. Ciupitu and I. Simionescu, "Optimal location of robot base with respect to the application positions," in Proc. of the 2-nd International Conference on "Optimization of the Robots and Manipulators" OPTIROB 2007, pp. 57-62, May 27-29, 2006.

[19] L. Ciupitu, S. Brotacand, and S. Chivescu, "Optimum position of an industrial robot used in forge applications," in Proc. of the 4-thInternational Conference on "Optimization of the Robots and Manipulators", A. Olaru, L. Ciupitu, S. Olaru, and Constanta, Ed. Mamaia, Romania, 28th-31st, 2009, pp. 43-47.

[20] J. Denavit and R. S. Hartenberg, A kinematic notation for lower-pair mechanisms based on matrices, Trans. of ASME, Journal of Applied Mechanics, vol. 23, pp. 215-221.
[21] J. T. Feddema, "Kinematically optimal placement for minimum time coordinated motion, robotics and automation," IEEE International Conference, vol. 4, no. 22-28, pp. 3395-3400, Apr. 1996.

[22] F. Kovacs and G. Cojocaru, Manipulatoare, roboțI şI aplicaţiile lor industriale, EdituraFacla, Timişoara, 1982.

[23] I. Simionescu, V. Moise and L. Ciupitu, "Sintezafuncţiilor de transmitere ale mecanismelor," Journal of Romanian Academy "Studii și cercetări de mecanică aplicată”, vol. 55, no. 5-6, pp. 409-426, 1996.

[24] I. Simionescu and L. Ciupitu, "On the optimisation of industrial robot motions," T.C.M.M. Journal of EdituraTehnică, nr. 28 (special number with the Proceedings of the Seventh IFToMM International Symposium on Linkages and Computer Aided Design Methods - Theory and Practice of Mechanisms - SYROM '97), vol. 2, pp. 333-338, 1997.

[25] I. Simionescu and L. Ciupitu, Optimum programming of industrial robot trajectories, in Proc. of the 3-rd Asian Conference on Robotics and Its Application, pp. 219-224, October 29- 30, 1997.

[26] L. Tian and C. Collins, "Optimal placement of a two-link planar manipulator using a genetic algorithm," Robotica Journal, vol. 23, no. 2, Cambridge University Press, Mar. 2005, pp. 169-176.

[27] A. Olaru, S. Olaru, and N. Mihai, "Proper assisted research method solving of the robotsinverse kinematics problem," Applied Mechanics and Materials, vol. 555, pp. 135-147, 2014.

[28] A. Olaru, S. Olaru, and L. Ciupitu, "Assisted research of the neural network by back propagation algorithm," OPTIROB 2010 International Conference, Calimanesti, Romania: The Reserch Publishing Services Singapore Book, 2010, pp. 194-200.

[29] A. Olaru, S. Olaru, and N. Mihai, "Proper assisted research method solving of the robots inverse kinematics problem," Applied Mechanics and Materials, vol. 555, 135-147, 2014.

[30] A. Olaru, S. Olaru, and L. Ciupitu, "Assisted research of the neural network by back propagation algorithm," OPTIROB 2010 International Conference,Calimanesti, A. Olaru, L. Ciupitu, and S. Olaru, Ed. Romania: The Research Publishing Services Singapore Book, May 28th-30th, 2010, pp. 194-200.

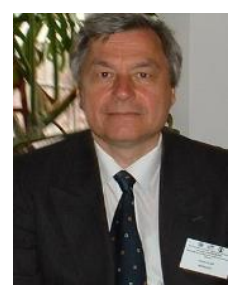

Adrian Olaru finished the University Politehnica of Bucharest, the Faculty of Machine-Tools, Machine and Manufacturing Systems Department. Now, from 1998 he is a university full professor, and he teaches the following courses: industrial robots dynamics behavior, LabVIEW application in modeling and simulation of the dynamic behavior of robots and personal and social robots. He is a doctor from 1989. In the last ten years he has been leading the following research projects: computer aided research and design for the hydraulic amplifiers of pneumatic and hydraulic screwdrivers; computer aided research over the dynamic behavior of the forging manipulator orientation modulus; computer aided research over dynamic behavior of the charging manipulators tipping modulus; computer aided research over dynamic behavior of the charging manipulators translation modulus; experimental validation for mathematical models of hydraulic elements and servo system; methodological guide for dimensioning and optimizing electrohydraulic elements; design of the mobile robots; assisted research of the magneto rheological dampers; assisted research of the intelligent dampers; assisted research of the neural networks; optimizing of the robots dynamic behavior by using the Fourier proper analyzer; optimizing the dynamic compliance and global transmisibility by using the assisted research and proper LabVIEW instrumentation; optimize the dynamic behavior and the space trajectory by using the proper neural network.

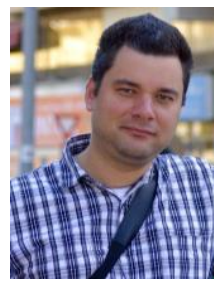

Serban Olaru finished the University Politehnica of Bucharest, Faculty of Machines and Manufacturing Systems, Romania. From 2008 he become the Ph.D.Eng.in the field of mechatronics. Now, he works in RomSYS private company, from Bucharest, Romania, in the department of mechatronics. He write more than 50 research papers in the fields of intelligent damper systems, mechatronic systems, simulation and modeling with LabVIEW instrumentation.

Mihai C. Niculae finished the University Politehnica of Bucharest, Faculty of Machines and Manufacturing Systems, Romania. From 2006 he become Ph.D.Eng. in the field of robotics. Now, he is the manager of the private company in mechatronics systems, Technoaccord, Quebec, Canada. 